\title{
Opportunities and Challenges of Global Mental Health
}

\author{
Richard Balon ${ }^{1} \cdot$ Eugene V. Beresin ${ }^{2} \cdot$ Adam M. Brenner $^{3} \cdot$ John H. Coverdale ${ }^{4}$. \\ Anthony P. S. Guerrero ${ }^{5}$ Alan K. Louie ${ }^{6}$. Laura Weiss Roberts ${ }^{6}$
}

Received: 9 May 2016 / Accepted: 10 May 2016/Published online: 6 June 2016

(C) Academic Psychiatry 2016

Global mental health has been defined as an area of study, research, training, and practice that places a priority on improving and achieving equity in mental health for all people worldwide [1, 2]. Global mental health has come to the forefront of discussions and deliberations among policy thinkers and educators during the last decade. It has become obvious that mental health, or rather mental illnesses and related disabilities, is a global issue. It is also clear that mental health cannot be disentangled from overall health. Prince and colleagues [3], in their aptly titled article "No Health Without Mental Health," pointed out that about $14 \%$ of the global burden of disease has been attributed to neuropsychiatric disorders. They also warned, however, that the burden of mental disorders is likely to be underestimated because of the limited appreciation of the connectedness between mental illness and other diseases. As they wrote, mental disorders increase the risk of communicable and noncommunicable diseases and injuries and many other medical illnesses increase the risk of mental disorders.

Patel and Prince [2] pointed out the disparity in resources among countries around the world - they report that low- and middle-income countries are home to more than $80 \%$ of the

Richard Balon

rbalon@wayne.edu

Wayne State University, Detroit, MI, USA

2 Harvard Medical School, Boston, MA, USA

3 University of Texas Southwestern Medical Center, Dallas, TX, USA

4 Baylor College of Medicine, Houston, TX, USA

5 University of Hawai'i John A. Burns School of Medicine, Honolulu, HI, USA

6 Stanford University, Stanford, CA, USA global population but command less than $20 \%$ of the share of the mental health resources. The more concrete and detailed numbers are even more staggering. In 2007, Saxena and colleagues [4] noted serious shortages of psychiatrists in some countries. For example, Chad, Eritrea, and Liberia had one psychiatrist each (respective populations 9, 4.2, and 3.5 million), and Afghanistan, Rwanda, and Togo had just two psychiatrists each (populations 25, 8.5, and 5 million). Lowincome countries had a median of 0.05 psychiatrists and 0.16 psychiatric nurses per 100,000 population. Highincome countries had a ratio of psychiatric health care workers to population about 200 times higher [4]. The disparity is not only in the mental health workforce but also in the personal financial resources available for health care and in systemic financing of mental health care and treatment of mental illnesses.

Almost a decade ago, Horton [5] wrote that although the World Health Organization (WHO) has continued to publish reports on mental health, "somehow the agency, through its leadership and partnerships, has been unable to convert fine words into tangible actions at country level. Partly this is because WHO has not backed its words with resources. And partly it is because WHO's leadership has failed to build a sustainable mechanism across global and country institutions to hold itself and others accountable for its recommendations. This paralysis is surprising. Many low-income countries and civil society groups are crying out for help" ([5], p. 806). Horton pointed out that other organizations (e.g., The World Bank) have also done very little in relation to the level of influence they could have in changing awareness and resources to promote mental health throughout the world.

This disturbing situation has not changed substantially over the last decade. However, academic departments of psychiatry have been an exception to this pattern of relative neglect and inaction in relation to global mental health needs. Multiple 
initiatives and programs fostering education, training, and research have been developed around the world, as documented in this issue of Academic Psychiatry.

\section{Opportunities}

Patel [6] pointed out that global mental health initiatives provide numerous opportunities. They have the potential to contribute to "discovery science": "Given the complex nature of mental disorders, where multiple social, genetic, biological and environmental determinants interact to lead to psychopathology, it is plausible that studying these disorders, using similar protocols in diverse global populations, may yield new insights into aetiology of mental disorders. This is no small opportunity for decades of research focused on a small fraction of humanity has yet to yield any findings which have led to a transformational advance in our understanding of the nature of mental disorders. A similar promise could be applied to the area of therapeutics" ([6], p. 2). Patel [6] also suggests several areas where the general and research initiatives of global mental health provide enormous opportunities to generate knowledge with global application: epidemiological research that seeks to better characterize the nature of mental disorders, possibly in various cultural contexts; promotion of novel interventions, namely, psychosocial ones (e.g., mindfulnessbased therapy, with its origins in Buddhism and Hinduism) and herbal and physical remedies used in traditional medicines (e.g., Chinese medicine, Ayurveda); and investigation of the co-existence of mental and other medical disorders.

We have already appreciated some important inroads that psychiatry has made to advancements in global mental health research. For example, Becker and colleagues [7, 8] have studied the impact of media on body image in adolescent females in Fiji longitudinally and, more recently, studied means of identifying adolescents at psychiatric risk in a school-based screening. Borba and colleagues [9] have studied the origins and presentation of posttraumatic stress disorder in Liberian youth as an outcome of years of war. Loar et al. [10] studied the risks and protective factors facing children in displacement during the Gulf War. Projects such as these not only shed insight into global mental health but also provide opportunities for psychiatry residents and psychology trainees to develop qualitative and quantitative research skills, as well as skills to diagnose and treat psychiatric disorders in a wide range of cultural groups. They provide trainees with tools needed to address mental health disparities that clearly exist even in high-income countries.

Beyond the opportunities for psychiatric global research and intervention, academic institutions also offer opportunities for residents and junior faculty to work within multidisciplinary teams around the world and help to develop new and sustainable programs in low- and middle-income countries. Not only are residents working with teams remotely, but they have an opportunity to train local health care professionals to assess and treat psychiatric disorders. One example of this mission is the Toronto Addis Ababa Psychiatry Project [11], which created a remarkable longitudinal partnership between an Ethiopian psychiatry residency training program and the University of Toronto faculty and residents. Another example is the Partners in Health intervention in Haiti following the 2010 earthquake [12]. In this intervention, a psychiatric resident became a core team member with the explicit mission to educate local physicians in the manifestations of psychiatric disorders. One of the oldest collaborative programs was started by the University of Hawaii during the 1970s to introduce child and adolescent psychiatry to Indonesia [13]. The program was renewed and modernized (e.g., by using videoconferencing) in 2009. Such missions are extraordinary experiences for residents and assist to offset the terrible shortage of mental health professionals by informing non-psychiatric professionals about the signs, symptoms, and treatment of mental illness.

\section{Challenges}

Collins and colleagues [14] identified 25 grand challenges in global mental health and grouped them into six goals: to "identify root causes, risk and protective factors; advance prevention and implementation of early interventions; improve treatments and expand access to care; raise awareness of the global burden; build human resource capacity; and transform healthsystem and policy responses." Psychiatric educators could play roles in all these areas, especially in building human resource capacity and raising awareness of global burden. Examples of specific challenges in building human resources include increasing capacity in low- and middle-income countries through partnerships with organizations in high-income countries to create regional centers for mental health research, education, training, and practice that incorporates the views and needs of local people; developing sustainable models and programs to train and increase the number of culturally and ethnically diverse lay and specialist providers to deliver evidence-based services; and strengthening the mental health component in educating and training all health care personnel [15].

These challenges may be even more acute today, given the ongoing crisis of refugees fleeing from civil wars, human rights atrocities including human trafficking, and economic hardship worldwide [16, 17]. Human labor and sex trafficking, for example, is a major global health problem [17] about which little has been done in the way of development of formal educational curricula [18]. Refugee populations suffer from a profound increased risk of mental illness due to at least two distinct types of stress [19, 20]. First, many have suffered significant traumatic exposures. A partial list would include threat of death during combat or collateral destruction, sexual 
assault during war or during later flight, witnessing death and horrific injuries, threat or experience of torture, and homelessness. Second, refugee communities suffer from acculturative stress, including the pressures to assimilate, separation from family, discrimination in their host countries, and intergenerational conflicts. Some academic psychiatry departments have risen to this challenge with innovative clinics and programs that focus on refugee mental health and offer valuable training opportunities [21,22].

Another major challenge is the view that the global mental health initiatives are a "neocolonial project to globalize western psychiatry" [23]. Given the significant history of Western colonialism well into the 20th century and the numerous violent political reactions it engendered, this is a perspective that must be responded to - respectfully and proactively. The limits of evidence derived from studies in high-income countries when applied to low- and middle-income countries must also be recognized. For example, evidence on preventing violence against women and girls is highly skewed toward that from high-income countries [24]. Having an understanding of local culture and language skills, therefore, should be part of any high-income country's initiative for global mental health in low- and middle-income countries, and every effort should be made to partner with local health care systems and providers in studying mental illness and mutually developing interventions that will fit with the local ecology.

Some of these goals and challenges are already being addressed, as noted earlier. Psychiatric education seems to be rising to the challenge to help build a better and functional global mental health. We need to become routinely sensitive to different cultural perspectives, to unmet global mental health needs, and to barriers to mental health services in specific global settings, including stigma and discrimination [24].

\section{What Could Be Done?}

The major work in global mental health definitely lies ahead. The educational, research, and clinical intervention initiatives that have been created or planned are just first steps in the right direction. We need to build on these efforts, showing leadership across medicine and society more broadly to foster not only awareness but action. We need to advocate for transforming health-system and policy responses, integrating mental health into primary care in low- and middle-income countries, decentralizing mental health service, increasing financial support, and strengthening public health perspectives in mental health [3]. In doing this work, however, we must show humility and authentic regard for our international partners, who often have developed sound local solutions in support of mental health - and often under the direst circumstances. We have much to learn in this process, and we must remain mindful that our high-cost, specialist-oriented, and resource-intensive systems of care may not be suited to much of the world. Indeed, many countries have better health outcomes for physical disorders that exist in the USA and Canada; exploring how different countries approach mental health is an important role for academic psychiatry. As Patel and Prince [2] wrote, "Knowledge can and must flow in both directions between high-income countries and low- and middle-income countries" (p. 1977).

Academic psychiatry has the potential to play a major role in gathering information about mental health around the world and in understanding rapidly evolving global mental health needs. By full understanding of all aspects of global mental health, we will be able to avoid the risk that global mental health becomes a solution without a clearly defined problem. We may help generate new knowledge, and, through methods of implementation science and novel technology, we may help drive change through academic-community partnerships [25]. We are positioned to help in truly transforming global mental health. More than 100 years ago, Florence Nightingale said, "I think one's feelings waste themselves in words; they ought all to be distilled into actions which bring results." In light of the gravity of disability and premature death associated with mental disorders throughout the world, we could not agree more.

\section{Compliance with Ethical Standards}

Disclosure On behalf of all authors, the corresponding author states that there are no conflicts of interest.

\section{References}

1. Koplan JP, Bond TC, Merson MH, et al. Towards a common definition of global health. Lancet. 2009;373:1993-5.

2. Patel V, Prince M. Global mental health: a new global health field comes of age. JAMA. 2010;303:1976-7.

3. Prince M, Patel V, Saxena S, et al. No health without mental health. Lancet. 2007;370:359-77.

4. Saxena S, Thornicroft G, Knapp M, Whiteford H. Resources for mental health: scarcity, inequity, and inefficiency. Lancet. 2007;370:878-89.

5. Horton R. Launching a new movement for mental health. Lancet. 2007;370:806.

6. Patel V. From delivery science to discovery science: realising the full potential of global mental health. Epidemiol Psychiatr Sci. 2016. doi:10.1017/S2045796016000263.

7. Becker AE, Burwell RA, Gilman SE, Herzog DB, Hamburg P. Eating behaviours and attitudes following prolonged exposure to television among ethnic Fijian adolescent girls. Br J Psychiatry. 2002;180:509-14

8. Becker AE, Bainivualiku BA, Khan N, et al. Feasibility of a schoolbased behavioral health risk study in an ethnic Fijan adolescent female study population in Fiji: the HEALTHY Fiji study. Fiji Med J. 2009;28:18-34.

9. Borba $\mathrm{CP}, \mathrm{Ng} \mathrm{LC}$, Stevenson A, et al. A mental health needs assessment of children and adolescents in post-conflict Liberia: results from a quantitative key-informant survey. Int J Cult Ment Health. 2016;9:56-60. 
10. Laor N, Wolmer L, Mayes LC, Gershon A, Weizman R, Cohen DJ. Israeli preschool children under Scuds: a 30-month follow-up. J Am Acad Child Adolesc Psychiatry. 1997;36:349-56.

11. Alem A, Pain C, Araya M, Hodges BD. Co-creating a psychiatric resident program with Ethiopians, for Ethiopians, in Ethiopia; the Toronto Addis Ababa Psychiatry Project (TAAPP). Acad Psychiatry. 2010;34:424-32.

12. Grelotti DJ, Lee AC, Fils-Aimé JR, et al. A pilot initiative to deliver community-based psychiatric services in rural Haiti after the 2010 earthquake. Ann Glob Health. 2015;81:718-24.

13. Guerrero A, Wiguna T, McDermott J. The University of Hawaii/ University of Indonesia collaboration to build and sustain a child psychiatric workforce. Acad Psychiatry. 2014;38:209-12.

14. Collins PY, Patel V, Joestl SS, et al. Grand challenges in global mental health. Nature. 2011;475:27-30.

15. Fricchione GL, Borba CP, Alem A, Shibre T, Carney JR, Henderson DC. Capacity building in global mental health: professional training. Harv Rev Psychiatry. 2012;20:47-57.

16. Gostin LO, Roberts AE. Forced migration: the human face of a health crisis. JAMA. 2015;314:2125-6.

17. Walk Free Foundation. The global slavery index 2014. Available at www.globalslaveryindex.org. Accessed 05/01/2016.

18. Coverdale J, Beresin EV, Louie AK, Balon R, Roberts LW. Human trafficking and psychiatric education: a call to action. Acad Psychiatry. 2016;40:119-23.
19. Hwang WC, Myers H, Abe-Kim J, Ting J. A conceptual paradigm for understanding culture's impact on mental health: the Cultural Influences on Mental Health (CIMH) Model. Clin Psychol Rev. 2008;28:211-27.

20. Fazel M, Reed RV, Panter-Brick C, Stein A. Mental health of displaced and refugee children resettled in high-income countries: risk and protective factors. Lancet. 2012;379: 266-82.

21. Pumariega AJ, Rothe E, Pumariega JB. Mental health of immigrants and refugees. Community Ment Health J. 2005;41:581-97.

22. Boehnlein JK, Leung PK, Kinzie JD. Cross-cultural psychiatric residency training: the Oregon experience. Acad Psychiatry. 2008;32:299-305.

23. Lamichhane J. Q\&A with China Mills, Researcher, Oxford Poverty and Human Development. Movement for Global Mental Health Newsletter, May 2014. Available at http://www. globalmentalhealth.org/newsletter_archive/newsletter-may. Accessed 7 May 2016.

24. Coverdale J, Roberts LW. Global challenges and ethics in protecting and promoting the interests of psychiatrically ill patients. Int Rev Psychiatry. 2010;22:229-34.

25. Roberts LW, Reicherter D, Adelsheim S, Joshi SV. Partnerships for mental health: narratives of community and academic collaboration. New York: Springer Science + Business Media, LLC; 2015. 